\title{
Promoting Bone Health in MPS VI (Maroteaux-Lamy syndrome): Framing New Therapies, Part 1
}

In October 2008, more than 80 clinicians and scientists gathered at the Children's Hospital Oakland Research Institute in Oakland, California, at a two day meeting of experts entitled "Promoting Bone Health in MPS VI: Framing New Therapies". The meeting was jointly sponsored by Children's Hospital and Research Center Oakland and the Department of Pediatrics, University of Padova, Italy. The meeting, including travel stipends and honoraria for speakers, was supported by an educational grant from BioMarin Pharmaceutical Inc., Novato, CA.

Mucopolysaccharidosis (MPS) VI is a rare autosomal recessive genetic disorder involving mutation and abnormal function of the lysosomal enzyme $N$-acetylgalactosamine 4-sulfatase (arylsulfatase B; ASB). Decreased enzyme activity leads to incomplete degradation of the glycosaminoglycans (GAG) dermatan and chondroitin 4 sulfate, and accumulation of breakdown products in cells and tissues. These breakdown products contribute to lysosome damage, cell death, and organ dysfunction. Although a wide spectrum of clinical severity occurs in many organ systems, the predominant findings are related to a skeletal dysplasia including short stature, dysostosis multiplex, and joint disease. Until recently, treatment involved primarily supportive medical and surgical care. Successful hematopoietic stem cell transplantation (HSCT) has been described in a few case reports for MPS VI although the risk associated with transplantation is significant and obtaining compatible donors is difficult. In 2005, Naglazyme ${ }^{\circledR}$ (galsulfase; recombinant human $N$-acetylgalactosamine 4-sulfatase, rhASB) was approved by the Food and Drug Administration (FDA) as an intravenous enzyme replacement therapy (ERT) for MPS VI. With the introduction of this new therapy, it has become imperative for us to improve our basic understanding of the underlying bone and joint disease, effects of specific enzyme therapy on bone and joint disease and the importance of improving management techniques. Although this conference was intended to focus on MPS VI, it was apparent that the spectrum of bone and joint disease and possible underlying pathologic and molecular mechanisms, as well as available specific and supportive therapies, could be generalized to other mucopolysaccharide diseases, in particular MPS I (Hurler, Hurler-Scheie, Scheie syndromes) and MPS II (Hunter syndrome). Many of the speakers have included references or even focused their presentations and manuscripts on these other MPS diseases; particularly if the available research has primarily involved these diseases.

The program developed by guest editors, Paul Harmatz, Joseph Muenzer, and Maurizio Scarpa, included a diverse group of 16 speakers covering a broad range of topics important to bone health in MPS VI patients including basic bone and cartilage biology, radiologic evaluation of bone health and disease, growth and endocrine therapy to improve bone growth in MPS patients, orthopedic and neurosurgical problems and management, emergence of new therapies such as intravenous, intrathecal, intra-articular enzyme replacement therapy, and finally mechanisms of glycosaminoglycan-mediated joint inflammation and damage and possible anti-inflammatory therapies. Finally, a number of the clinicians presented case reports that illustrated the broad range of issues surrounding treatment of bone disease in MPS patients. The speakers were invited to prepare and submit manuscripts 
to the Journal of Pediatric Rehabilitation Medicine (JPRM) for publication with two issues to be focused on this topic. All manuscripts received external expert peer-review with reviewers blind to the individual authors submitting each manuscript.

The guest editors hope that the interaction of clinicians and basic scientists will help stimulate productive collaborations; provide basic scientists with new areas to explore basic mechanisms of bone growth and disease in MPS; broaden radiology and surgical consultants' understanding of MPS disease and focus their interest in developing expertise in MPS clinical management; and ultimately help pediatricians and geneticists better understand the underlying basic science impacting bone disease in MPS and the range of approaches available to radiologists and surgeons.

Finally, we gratefully acknowledge the financial support by BioMarin that enabled the guest editors to assemble this large group of experts and develop the manuscripts that constitute two focused issues of JPRM. We also acknowledge editorial assistance pro- vided by Ismar Healthcare NV, Belgium, and paid for by BioMarin Pharmaceutical Inc., Novato, CA, on selected manuscripts and acknowledged as part of each manuscript. BioMarin reviewed selected manuscripts to insure the accuracy of all statements regarding enzyme replacement therapy with galsulfase, but had no role in the content presented and discussed at the meeting.

Guest Editors

Paul Harmatz

Children's Hospital \& Research Center Oakland,

Oakland, CA, USA

Joseph Muenzer

University of North Carolina at Chapel Hill, Chapel Hill, NC, USA

Maurizio Scarpa

Department of Pediatrics, University of Padova, Italy 